\title{
The psychometrics and diagnostic properties of the Montreal Cognitive Assessment 5-min protocol in screening for Mild Cognitive Impairment and dementia among older adults in Tanzania: A validation study
}

Golden Mwakibo Masika ( $\square$ golden.masika@link.cuhk.edu.hk)

The University of Dodoma College of Health Sciences https://orcid.org/0000-0003-0581-5797

Doris S.F. Yu

Chinese University of Hong Kong

Polly W.C. Li

Chinese University of Hong Kong

\section{Adrian Wong}

Chinese University of Hong Kong

Rose S.Y. Lin

Chinese University of Hong Kong

\section{Research article}

Keywords: MoCA-5-minutes protocol, cognitive impairment, psychometrics, Tanzania, mild cognitive impairment, neuropsychology

Posted Date: August 15th, 2019

DOI: https://doi.org/10.21203/rs.2.12943/v1

License: (c) (i) This work is licensed under a Creative Commons Attribution 4.0 International License. Read Full License

Version of Record: A version of this preprint was published at International Journal of Older People Nursing on September 13th, 2020. See the published version at https://doi.org/10.1111/opn.12348. 


\section{Abstract}

Introduction The prevalence of dementia in Tanzania, as in other developing countries is progressively increasing. Yet international screening instruments for mild cognitive impairment are lacking. The aim of this study was to determine the psychometrics and the diagnostic ability of the Montreal Cognitive Assessment 5 minutes protocol (MoCA-5-min) among older adult in the rural Tanzania. Methods The MoCA-5-min and the IDEA cognitive screening were concurrently administered through face to face to 202 community-dwelling older adults in Chamwino district. Exploratory factor analysis (EFA) using principal component method and oblique rotation was performed to determine the underlying factor structure of the scale. The concurrent, construct as well as predictive validities of the MoCA-5-min were examined by comparing its score with IDEA cognitive screening and psychiatrist's diagnosis using DSM-V criteria respectively. Results The EFA found that all the MoCA-5-min items highly loaded into one component, with factor loading ranging from 0.550 to 0.879 . The intraclass correlation coefficient for 6 weeks test-retest reliability was 0.85 . Its strong significant correlation with the IDEA screening (Pearson's $r=0.614, p<0.001$ ) demonstrated a good concurrent validity. Using the psychiatrist's rating as the gold standard, MoCA-5-min demonstrated the optimal cut-off score for $\mathrm{MCl}$ at 22, which yielded the sensitivity of $80 \%$ and specificity of $74 \%$; and dementia at score of 16 giving a sensitivity of $90 \%$ and specificity of $80 \%$. Upon stratifying the sample into different age groups, the optimal cut-off scores tended to decrease with the increase in age. Conclusion The MoCA-5-min is reliable and provides a valid and accurate measure of cognitive decline among older population in the rural settings of Tanzania. The use of varying cut-off scores across age groups may ensure more precise discriminatory power of the MoCA-5-min.

\section{Introduction}

Dementia is a public health concern which currently affects 50 million people worldwide. In about 3 decades ahead the prevalence will triple to 152 million people impacting significantly to the quality of life and economy of the affected people, families and health care system [1, 2]. The impact could be much more worse in the low income countries such as Tanzania, where despite the greater predicted increase in the number of people affected, the level of preparedness is particularly poor, characterized by shortage of specialist doctors and nurses and inadequate medical resources to treat patients with dementia [3, 4]. To counter this effect, the World Health Organization (WHO) recommends measures to be taken to control the risk factors and prevent further cognitive decline among those who are at pre-dementia stages [5].

Mild cognitive impairment $(\mathrm{MCl})$ has become the more important stage for prevention of further cognitive decline because it is reversible [6], and the affected persons still maintain the functional ability to participate in treatments $[7,8]$. Nevertheless, the definitive and standard approach to identify $\mathrm{MCl}$ and/or dementia is burdensome and can be a barrier for early initiation of treatments for prevention of further decline [9]. The clinical procedures require the diagnosis to be performed by neurologists, and sometimes be accompanied with some laboratory and/or neuroimaging assessments, which could be expensive and non-feasible in low income countries [7]. To address this gap, many brief cognitive assessments have been developed and can be administered by trained personnel to identify the suspected cases among the high-risk population cohort for further formal diagnostic procedures [10]. Such step-down care has been commented as highly cost-saving initiative to promote better case identification for prompt care [11]. 
In Tanzania, brief instruments for screening $\mathrm{MCl}$ are lacking. In the past 7 years, a research group namely Identification and Intervention for Dementia in Elderly Africans (IDEA) has published many research articles related to dementia based in Hai district, northern Tanzania, but only one study focused on screening for $\mathrm{MCl}$ [12]. In that study, $\mathrm{MCl}$ screening was done using Cognitive Screening Instrument for Dementia (CSI-D) [13]. However, the validity of this tool for $\mathrm{MCl}$ screening among the older population in the rural Tanzania is questionable because it was validated in Kenya [12] where the Swahili language is spoken in a different dialect and the culture is slightly different [14]. Even the new diagnostic test for cognitive decline has emerged, such as the Six Items Dementia Screening for Africa (SIDSA), its validity in $\mathrm{MCl}$ screening has never been tested $[15,16]$. Indeed, none of the available tool in Africa is used internationally to facilitate more reliable cross-country comparison. To address the WHO's advocacy for dementia prevention and early detection, a reliable, valid and internationally common brief screening instrument for $\mathrm{MCl}$ is urgently needed.

The Montreal Cognitive Assessment is a psychometrically sound instrument available in nearly 100 languages for $\mathrm{MCl}$ screening. Although the Kiswahili-MoCA version is available for use in Tanzania, its cultural and contextual relevance is in question especially for its use in the rural area. This is because some of the items such as trail making, cube drawing, clock drawing, and verbal fluency test are not applicable to the illiterate responders, same as the case in Colombia study [17]. Recently, a briefer version of MoCA, namely MoCA-5-min protocol has been developed in Chinese language to facilitate a more time-efficient and education-bias free screening in Hong Kong. This briefer version was also designed to serve as the screening tool to be used in busy clinical settings and over the telephone to persons with neuro-vascular impairments such as stroke and transient ischemic accident. Four subsets were carefully selected from the original version to ensure it is content relevant to neurovascular impairment, brief and feasible to be administered via telephone and to those who are illiterate or with low education level. These items are conceptually adequate to assess five domains including attention, verbal learning and memory, executive functions/language and orientation [18]. Compared to the full version of MoCA, the MoCA-5-min protocol excluded the tasks requiring participants to draw, write, or attempt the phonemic fluency tests which could be less feasible for illiterate responders [17]. Given these characteristics, the MoCA-5min might be particularly content relevant to the people in rural Tanzania. Moreover, previous studies have documented the variability across populations with different education levels, which imply different cut-off scores should be used according to the level of education. Therefore, this study aimed to translate the MoCA-5-min to Kiswahili language, evaluate its psychometric and diagnostic properties by comparing its discriminative power for $\mathrm{MCl}$ and dementia with the IDEA cognitive screening against the psychiatrist's rating using Diagnostic and Statistical Manual of Mental Disorders, 5th Edition (DSM-V) criteria, and to determine the optimal cut-off scores.

\section{Methods}

This study received an ethics approval by the University of Dodoma Research Ethics Committee (Ref: UDOM/DRP/REC/63/). The study adopted a methodological approach to translate the MoCA-5-min and evaluate for its psychometric characteristics among the older adults in Chamwino district central Tanzania. The diagnostic accuracy against the psychiatrist's rating of $\mathrm{MCl}$ and dementia was also compared with that of the IDEA cognitive screening. Multiple steps were followed to validate the translated instrument. Firstly, an exploratory factor analysis (EFA) was performed to identify the underlying factor structure of the scale. Cronbach's alpha and item analysis were performed to determine the internal consistency of the MoCA-5-min. A test-retest reliability over a six-week period was computed. Concurrent validity was examined by identifying its correlation with the IDEA cognitive screening. Construct validity was evaluated by examining whether the MoCA-5-min was related to the 
comparing constructs including age, education, depressed mood, mental health and functional status on instrumental activities of daily living in the relationships as suggested by literature [19, 20]. The diagnostic accuracy of the MoCA-5-min against the psychiatrist's neurocognitive diagnosis rated by using DSM-V criteria was also examined.

\section{Translation and cultural modifications of the instrument}

A two-phase translation process was followed. First, the original Chinese version of the MoCA-5-min was translated to English by two independent translators, both fluent in English and Chinese. The two versions were harmonised through face to face discussion between the two translators to ensure agreement in the concepts and meanings of each item. The original developer of the instrument who is also fluent in both English and Chinese validated the conceptual and semantic equivalency of the English translated version in accordance with the original Chinese version [21].

Second, a forward and backward translation approach as recommended by Brislin [22, 23] was followed to translate the English version of the MoCA-5-min to Kiswahili version. Two independent bilingual individuals who are experienced in dementia research translated the instrument to Kiswahili language. One version of the translated tool was created upon discussion between the two translators to resolve the discrepancies and agree on the conceptual and semantic equivalency with the English version. There was no cultural modification of the items because all items were deemed relevant to the Tanzania older population except for four words in the multiple-choice list, including 'demin', 'velvet', 'daisy' and 'tulip'. The lack of cultural relevance is mainly because these textile materials and flowers were uncommon or not available in Tanzania. Upon discussion among the translators and the research team, the textile materials including "linen", "wool", "sunflower" and "lily" were used instead. All these items have similar popularity as the items in the original version for the Tanzania population.

Two registered psychiatrists who had been involved in dementia research validated the Swahili version of the instrument for cultural relevancy of the items. The final version of the instrument was translated back to English by the blinded bilingual translator. The back-translated version was compared with the original version and the identified discrepancies were used to make revision to the forward Kiswahili translated version to cross check if the conceptual meanings retained between the original English version and the Kiswahili version [23].

\section{Settings and Participants.}

The participants were recruited from six villages of the Chamwino district from January to February 2019 with convenience sampling. More than $80 \%$ of the older adults in Chamwino district live in the rural setting. The district comprise of about 24,154 older adults making $7.3 \%$ of the total population [24]. Eligible participants included those who were aged 60 or older, dwelling in the study villages, speaking Kiswahili, and consented to participate in the study. Those with severe physical illness or diagnosed with mental illnesses were excluded because of ethical reasons and/or possible bias of cognitive evaluation.

\section{Sample size estimation.}

Power analysis was used to determine the minimum sample size required for the multivariate analysis to examine the construct validity of the MoCA-5-min. As recommended by Anthoine et al, the computed number of subjects for bivariate correlation ( $r>0.2, a=0.05$ and $\beta=0.2)$ of the new and the reference scale to examine the concurrent validity was 200 [25]. As recommended by McNeil [26], the criterion validity using area under the 
receiver operating characteristics curves (AUC) analysis to predict the diagnostic accuracy, a minimum sample of 73 was needed with assumption that the level of significance was $5 \%$ and power as $80 \%$, at least $25 \%$ of the sample had $\mathrm{MCl}$ and the diagnostic accuracy of the MoCA-5-min to detect $\mathrm{MCl}$ would be moderate (i.e. $\mathrm{AUC}=$ $70 \%)$. This study recruited a total sample of 202 participants.

\section{Data collection procedure}

The research assistant approached the potential participants and conducted the screening against the eligibility criteria. After obtaining the written consent from the eligible participants, two cognitive tests including the MoCA5-min and the IDEA cognitive screening were administered to each participant by two trained research assistants. This was followed by administering the questionnaires for measuring the comparing constructs including depression and IADL. Thereafter, the participants were assessed by a registered psychiatrist, who was blinded from the cognitive test results, using the DSM-V criteria for neurocognitive evaluation [27]. Cognitive reassessment using MoCA-5-min was done to a subgroup of participants $(n=40)$ at six weeks later for assessing the test-retest reliability.

\section{Study instruments}

Montreal Cognitive Assessment 5- minutes protocol (MoCA-5-min): A 4-item tool which examines five cognitive domains: auditory attention, executive functions/language, orientation, verbal learning/encoding and memory. The scores range from 0 to 30, with higher scores indicating better cognitive function. The original Hong Kong version of the MoCA-5-min demonstrated good agreement $(r=0.87, p<0.001)$ with that of the full MoCA, and had an acceptable power to discriminate individuals with cognitive impairment from those without $(A U C=0.78)$ [18].

IDEA cognitive screening. A six-item cognitive assessment tool which measures orientation, verbal fluency, abstract reasoning, delayed recall and praxis. The scores range from 0 to 15 , and higher scores indicate better cognitive function [28]. The instrument was validated in Tanzania with good discriminatory power for dementia with AUC $=0.917$, and a sensitivity and specificity of $84.6 \%$, and $89.1 \%$ respectively at a cut-off score of $\leq 8[16]$.

Geriatric Depression Scale-15 items (GDS-SF): It was used to measure depressive symptoms. The instrument has 15 self-reported dichotomous items of YES or NO, with higher scores indicating more depressive symptoms, scores $>5$ suggest the presence of depression [29]. Our previous study in Tanzania (data will be published elsewhere) translated and validated the instrument and found the Cronbach's alpha was 0.81 and an excellent construct validity by showing a strong negative correlation with the Mental Health Inventory (MHI-5) $(r=-0.790, p$ $<0.001)$.

Mental Health Inventory-5 items (MHI-5): Is a brief screening questionnaire which assesses emotional well-being. It has 5 items to be rated on a scale ranging from 1 (all the time) to 6 (none of the time). As a subscale of the Short Form Health Survey (SF-36), MHI-5 was validated in Tanzania and demonstrated acceptable internal consistency (Cronbach's $\mathrm{a}=0.78$ ) and the correlation between $\mathrm{MHI}-5$ and the hypothesized scale was 0.55 with $95 \%$ scaling success rate [30].

Lawton Instrumental Activities of Daily Living (Lawton IADL): Is a questionnaire that measures functional ability in 8 domains of instrumental activities of daily living (IADL): telephone use, shopping, food preparation, housekeeping, laundry, mode of transportation, medication responsibility and handling finances. The scores range from 0 to 8 , with higher scores indicating better functional ability [31]. Our previous study translated and 
validated the instrument, the internal consistency was good (Cronbach's $a=0.81$ ) with item total correlation ranging from 0.36 to 0.69 . The instrument also demonstrated good concurrent validity with IDEA study Instrumental Activities of Daily Living (IDEA-IADL) questionnaire for measuring instrumental activities of daily living $(r=-0.593 p<0.001)$. (Data will be published elsewhere).

\section{Data analysis}

Analyses were performed using Statistical Package for Social Sciences version 25 [32]. Descriptive statistics were used to summarize socio-demographic characteristics of the sample. Internal consistency of the MoCA-5-min was determined by computing the Cronbach's alpha (a), item analysis and item-to total correlations [33, 34]. EFA was performed using the principal component method and oblique rotation [35] to determine the underlying factor structure of the scale. Further, the intraclass correlation coefficient (ICC) was computed to examine the six weeks test-retest reliability of the MoCA-5-min using 40 randomly selected participants.

As for the concurrent validity, the Pearson's correlation coefficient was used to examine the correlation of the MoCA-5-min scores with that of IDEA cognitive tests. Construct validity was examined by using multivariate regression to identify the independent relationships between the tested MoCA-5-min score and the hypothesized relating constructs including age, educational level, depression, psychological well-being and IADL function, as suggested by literature $[19,20]$. Variables which demonstrated bivariate relationship with MoCA-5-min at $p \leq 0.2$ were entered to the multivariate regression model. Finally, the receiver operating characteristics (ROC) analysis was performed to examine the discriminatory power of the MoCA-5-min against the psychiatrist's rating of the neurocognitive diagnosis, using the AUC $\leq 0.7$ defined as acceptable discriminatory power [26]. Then, the sensitivities, specificities and the probable cut-off scores of MoCA-5-min for detecting $\mathrm{MCl}$ and dementia was determined [36]. Its diagnostic accuracy was also compared with that of the IDEA cognitive screening.

\section{Results}

\section{Demographic characteristics of the sample}

A total of 202 participants were recruited from January to February 2019. Table 1 summarized the sample characteristics. Among this group, women were over-represented (72.3\%) and the mean age was 73.56 years (SD $=8.0$; median $=73.0$ ). Education level was fair; $46.5 \%$ were illiterate and less than one fifth of them had five years of education or above. Table 1 also lists the cognitive, psychological and functional characteristics of the participants. Comparison between gender indicated that the male participants were significantly older, with better education and cognitive function in terms of the MoCA-5-minute and IDEA cognitive scores.

\section{Internal structure and reliability of the MoCA-5-min}

The EFA revealed a single component with eigenvalue higher than one, which explained for $52.7 \%$ of variance, with the item loadings as 0.635 (auditory attention), 0.550 (verbal fluency/ language), 0.646 (orientation), 0.860 (free recall/encoding) and 0.879 (cued recall/memory). The Cronbach's alpha of MoCA-5-min was 0.74 with the corrected item to total correlations ranging from 0.36 to 0.75 . The Cronbach's alpha did not increase by more than 0.1 no matter which item was deleted, indicating items were homogeneous in measuring cognitive function. The intraclass correlation coefficient between baseline scores and the 6 weeks follow-up was 0.85 , suggesting MoCA5-min had high test-retest reliability. 
The MoCA-5-min demonstrated a strong positive correlation $(r=0.614, p<0.001)$ with the IDEA cognitive screening which suggested a good concurrent validity. As for the construct validity, Table 2 lists the results of the multi-variate analysis. Among the five hypothesized relating factors, only age $(r=-0.38, p<0.001)$; years of education ( $r=0.48, p<0.001)$, and Lawton IADL $(r=0.28, p<0.001)$ demonstrated a bivariate relationship with MoCA-5-min at $p \leq 0.2$. The multivariate analysis indicated that all these three factors were independently associated with the MoCA-5-min score in the hypothesized relationships, with level of education having the largest predictive power.

\section{Discriminatory power of the MoCA-5-min and IDEA for cognitive screening}

By using the DSM-V criteria, the psychiatrist diagnosed 156 participants ( $\mathrm{n}=156,77.2 \%$ ) as having $\mathrm{MCl}$, and the others were either cognitively normal $(n=36,17.8 \%)$ or having dementia $(n=10,5 \%)$. The MoCA-5-min scores were significantly different among these three diagnostic groups, and the post-hoc analysis indicated each group was significantly different from the others in the predicted direction (Table 3).

As for the diagnostic accuracy, MoCA-5-min demonstrated a good discriminatory power (AUC $=0.861,95 \% \mathrm{Cl}=$ $0.799-0.922$ ), which was better than that of the IDEA cognitive screening (AUC $=0.796,95 \%$ Confidence interval $(\mathrm{Cl})=0.727-0.865)$ (Figure 1). The optimal cut-off MOCA-5-min scores of 22 for $\mathrm{MCl}$ had a sensitivity of $80 \%$ and specificity of 74\%; whereas the optimal cut-off score of IDEA cognitive screening (i.e., score $=12.5$ ) gave a comparable sensitivity (70\%) and specificity (80\%).

In discriminating dementia, MoCA-5-min demonstrated an excellent discriminatory power $(\mathrm{AUC}=0.910,95 \% \mathrm{Cl}=$ $0.852-0.967)$, slightly better than that of IDEA cognitive screening (AUC $=0.859,95 \% \mathrm{Cl}=0.765-0.954)$ which also suggested that MoCA-5-min can better discriminate individuals with dementia from those with normal cognition (Figure 2). The cut-off MoCA-5-min score at 16 for dementia was optimal which yielded a sensitivity of $90 \%$ and specificity of $80 \%$; whereas the optimal cut-off score of IDEA cognitive screening (i.e., score =9) gave a lower sensitivity of $80 \%$ and specificity of $80 \%$.

After stratification of the sample into age groups and levels of education, we found that the diagnostic power was lower among the oldest old and the optimal cut-off scores were decreasing with an increasing age while maintaining the sensitivities and specificities; whereas, no significant change was noted across groups of educational levels in diagnostic power, optimal cut-off scores and their associated sensitivities. However, lack of normal cognitive controls in the illiterate group, precluded us from achieving a valid ROC analysis for this group (Table 4).

\section{Discussion}

The is the first study to examine the psychometric qualities and diagnostic accuracy of an international cognitive screening tool in the rural Tanzania. To minimize the threats to content relevancy resulting from the low educational background and unique cultural exposure of the older population in rural Tanzania, the 5-min version 
of the MoCA was adopted. The results indicated that the MoCA-5-item has high internal consistency and stability in measuring cognitive function as a single-dimension construct. Its significant correlations with the IDEA cognitive screening also suggested its good concurrent validity. Construct validity is evident even though the expected relationship with psychological variables was not identified. The most encouraging findings are that MoCA-5-min has high diagnostic accuracy in identifying dementia in both pre-clinical and clinical stage. This brief and cultural relevant screening tool not only supplement the limitation of existing cognitive screening tools available in Africa for detecting $\mathrm{MCl}$ and dementia, but also facilitate mapping the cognitive health of the Tanzanian population to an international reference.

Along with satisfactory reliability and validity that MoCA-5-min demonstrated, our study further demonstrated the excellent feasibility of using MoCA-5 for those older adults with no or very low level of education. These individuals were able to attempt all the items with the response rate of $100 \%$. Our previous study using the fullscale of MoCA in the same population showed that those with no or limited education were not able to attempt some of the items, such as trail making, cube drawing, clock drawing, and phonemic verbal fluency test (data to be published elsewhere). This observation is consistent with previous studies done in the population with similar sample characteristics [17], where participants who were illiterate or have very little education failed to respond to some items of the MoCA. Our findings found that MoCA-5-min is a relevant alternative for this population, it offers a reliable and valid way to estimate cognitive function among older adults in Tanzania.

With the known variability for different education levels age groups, the concept of "one cut-off never fits all" was introduced for using the MoCA-5-min by the authors, since a single cut-off score would highly misclassify those with less education and those with older age [37]. A similar performance trend was observed in the present study when the oldest old tended to be over diagnosed as cognitively impaired when an optimum cut-off score of 22/23 was used. However, decreasing the cut-off score with increasing age tended to maintain the sensitivity and specificity. This could be explained by the fact that, despite of no pathological cognitive impairment, the oldest old tend to score lower due to gradual accumulation of other pathologies and age related cognitive decline which could confound the diagnosis of cognitive impairment [38]. In such situation, diagnosis of cognitive impairment could be more precise if the age specific cut-off scores are to be implemented. Nevertheless, we were unable to uncover this pattern of findings for the subsample with illiterate group due to lack of adequate normal cognitive controls. However, this pattern of findings was not evident with the remaining educational groups which may imply that cognitive assessment using MoCA-5-min is not biased by education.

For establishing the construct validity, significant relationships were detected between MoCA-5-min and various comparing constructs, including age, education and functional ability. However, the relationships between MoCA5-min with depression and mental wellbeing were not significant, which was contradictory to the hypothesized relationships suggested by the available evidence $[39,40]$. While this phenomenon could be attributed to the overall good psychological functions of the sample, which might have rendered the relationships difficult to be detected; we also noted an unusual pattern of psychological response by majority of participants with poor cognitive functions who in fact, were mostly illiterate and economically poor. This group of participants tended to react to the questions of GDS-SF and MHI-5 more positively despite of obvious evidence of being deprived with health and social services/rights such as poor living conditions, multiple morbidities, poor access to medical care, and poverty [41]. This seemed to us that the group was unaware of the deprived health and social rights which lead to failure of gauging the value and quality of their lives which could impact to their psychological wellbeing. This phenomenon obscured the actual relationship of their psychological response pattern with cognitive 
function, unlike their counterparts with some educational background who would psychologically react accordingly when their health status, their economic and social wellbeing were not satisfactory. This pattern of findings could also be explained by the fact that, individuals with cognitive impairment who may have once experienced neuropsychological symptoms, be connected to faith healing and indoctrinations as found by the study in Hai district, Tanzania [4], who later would approach life in a more positive way even if one is stricken by the life difficulties.

\section{Implication for practice}

The fact that MoCA-5-min is identified in this study as having high diagnostic accuracy for both pre-clinical and clinical stages of dementia among the older population in Tanzania is highly important for improving detection and hence prevention and treatment of dementia in the country. The instrument can be utilized for rapid screening of cognitive function of older adults at risk of dementia in busy clinical settings and community settings by nonneurology trained staff and therefore offer opportunity for further diagnosis and initiation of early treatment or dementia prevention programs. The brevity and good psychometric and diagnostic properties of MoCA-5-min make it particularly suitable for this purpose.

Furthermore, by using MoCA-5-min to evaluate cognitive functions, facilitates the mapping of the cognitive health of the Tanzanian older population to an international reference which is highly recommended by WHO [5], and can encourage international collaboration of multicounty programs for dementia prevention. We therefore, recommend adoption of MoCA-5-min in screening for $\mathrm{MCl}$ and dementia in Tanzania.

\section{Limitations}

Some limitations are to be acknowledged in this study that would call for a cautious interpretation of the findings and recommendations for future studies. First, as this study was conducted in the rural settings, the findings may not be able to generalize to those living in urban areas with high level of education. Second, the convenience sampling method may also limit the generalizability. The sample included in our study was drawn from only one district in Dodoma region, which is only part of the geographically large Tanzania. Despite of many similarities of the older population in rural Tanzania, we must acknowledge that some cultural and economic variabilities that exist across the regions in Tanzania [42,43], which may further limit the generalizability of our findings. Third, our sample under-represented the male participants, which might be assumed to lead to gender bias in the results. However, since education and age which were significantly different across gender were independently accounted in the analysis, and whereas the findings did not show gender differences in the psychological and functional variables which are predictors of cognitive outcomes, we think that our conclusion may not be influenced by gender differences in the sample. We recommend further studies to be conducted in other regions of the country with random sampling to offer comparative evidence to our findings.

\section{Conclusions}

This study found that MoCA-5-min is reliable, valid and accurate in measuring cognitive function among older population in the rural settings of Tanzania. We also found that the use of different cut-off scores across age groups may improve the precision of discriminating cognitive impairment from normal cognition. 


\section{Declarations}

\section{Ethics approval and consent to participate}

Ethics approval was granted by the University of Dodoma Research Ethics Committee (Ref:

UDOM/DRP/REC/63/). All participants signed the informed consent forms.

\section{Consent for Publication}

NA

\section{Availability of data}

Data are accessible through the first the author. Researchers interested in further analysis are welcome to contact the first author upon securing ethics approval by the University of Dodoma, Tanzania

\section{Competing interests}

All authors declare no conflict of interest

\section{Funding}

This work was supported by the University of Dodoma (Grant no D02S03).

\section{Authros Contributions}

All authors provided a substantial intellectual contribution to the conception, data collections, analysis and drafting the manuscript. All authors read and approved the final manuscript for publication.

\section{Acknowledgement}

Ou reserch team wish to thank the Psychiatrist who worked with the first author in the communities and conducted the clinical diagnoses, the reserch assistants: Laidi Msisiri, Theresia Kessy and Paul Heady and the participants of the study.

\section{References}

1. World Health Organization. Dementia: Key facts. Fact Sheets. 2019; May:1-5. https://www.who.int/newsroom/fact-sheets/detail/dementia. Accessed 16 May 2019.

2. Ku LJE, Pai MC, Shih PY. Economic impact of dementia by disease severity: exploring the relationship between stage of dementia and cost of care in Taiwan. PLoS One. 2016;11:1-12.

3. Alzheimer's Disease International, Organization WH. Dementia: a public health priority. 2012. doi:978 924 1564458.

4. Hindley G, Kissima J, Oates LL, Paddick SM, Kisoli A, Brandsma C, et al. The role of traditional and faith healers in the treatment of dementia in Tanzania and the potential for collaboration with allopathic healthcare services. Age Ageing. 2017;46:130-7. 
5. World Health Organization World. First WHO ministerial conference on global action against dementia: Meeting report. Genever, Switzerland; 2015.

6. Sachdev PS, Lipnicki DM, Crawford J, Reppermund S, Kochan NA, Trollor JN, et al. Factors Predicting Reversion from Mild Cognitive Impairment to Normal Cognitive Functioning: A Population-Based Study. PLoS One. 2013;8:1-10.

7. Langa KM, Levine DA. The Diagnosis and Management of Mild Cognitive Impairment: A Clinical Review. J Am Med Assoc. 2014;312:2551-2561.

8. Rosebud R, Knopman DS. Classification and Epidemiology of MCI. Clin Geriatr Med. 2013;29:1-19.

9. Lussier M, Lavoie M, Giroux S, Consel C, Guay M, Macoir J, et al. Early detection of mild cognitive impairment with in-home monitoring technologies using functional measures: A systematic review. IEEE J Biomed Heal Informatics. 2018;2194 c:1-11.

10. Larner AJ. Cognitive screening instruments for the diagnosis of mild cognitive impairment. Prog Neurol Psychiatry. 2016;20 March:13-8.

11. Tong T, Thokala P, McMillan B, Ghosh R, Brazier J. Cost effectiveness of using cognitive screening tests for detecting dementia and mild cognitive impairment in primary care. Int J Geriatr Psychiatry. 2017;32:1392400.

12. Paddick S-M, Kisoli A, Samuel M, Higginson J, Gray WK, Dotchin CL, et al. Mild cognitive impairment in rural Tanzania: Prevalence, profile, and outcomes at 4-year follow-up. Am J Geriatr Psychiatry. 2015;23:950-9. doi:10.1016/j.jagp.2014.12.005.

13. Chen $\mathrm{C}-\mathrm{HH}$, Mizuno T, Elston R, Kariuki MM, Hall K, Unverzagt $\mathrm{F}$, et al. A comparative study to screen dementia and APOE genotypes in an ageing East African population. Neurobiol Aging. 2010;31:732-40. doi:10.1016/j.neurobiolaging.2008.06.014.A.

14. Harries L. The Nationalization of Swahili in Kenya. Lang Soc. 1976;5:153-64. https://www.jstor.org/stable/pdf/4166868.

15. Gray WK, Paddick SM, Collingwood C, Kisoli A, Mbowe G, Mkenda S, et al. Community validation of the IDEA study cognitive screen in rural Tanzania. Int J Geriatr Psychiatry. 2016;:1199-207.

16. Paddick SM, Gray WK, Ogunjimi L, Lwezuala B, Olakehinde O, Kisoli a, et al. Validation of the Identification and Intervention for Dementia in Elderly Africans (IDEA) cognitive screen in Nigeria and Tanzania. BMC Geriatr. 2015;15:53. doi:http://dx.doi.org/10.1186/s12877-015-0040-1.

17. Gõmez F, Zunzunegui M V., Lord C, Alvarado B, García A. Applicability of the MoCA-S test in populations with little education in Colombia. Int J Geriatr Psychiatry. 2013;28:813-20.

18. Wong A, Nyenhuis D, Black SE, Law LSN, Lo ESK, Kwan PWL, et al. The MoCA 5-min protocol is a brief, valid, reliable and feasible cognitive screen for telephone administration. Stroke. 2015;46:1059-64.

19. Baumgart M, Snyder HM, Carrillo MC, Fazio S, Kim H, Johns H. Summary of the evidence on modifiable risk factors for cognitive decline and dementia: A population-based perspective. Alzheimer's Dement. 2015;11:718-26. doi:10.1016/j.jalz.2015.05.016.

20. Alzheimer's Association. 2019 Alzheimer's disease facts and figures. Alzheimer's Dement. 2019;15:321-87. doi:10.1016/j.jalz.2016.03.001.

21. Van Widenfelt BM, Treffers PDA, De Beurs E, Siebelink BM, Koudijs E. Translation and cross-cultural adaptation of assessment instruments used in psychological research with children and families. Clin Child 
Fam Psychol Rev. 2005;8:135-47.

22. Brislin RW. Back translation for cross-cultural research. J Cross Cult Psychol. 1970;1:185-216.

23. Brislin RW. The wording and translation of research instruments. In: Field methods in cross-cultural research. 1986. p. 137-64.

24. National Bureau of Statistics. 2012 Population and Housing Census Population Distribution by Administrative areas. 2013.

25. Anthoine E, Moret L, Regnault A, Sbille V, Hardouin JB. Sample size used to validate a scale: A review of publications on newly-developed patient reported outcomes measures. Health Qual Life Outcomes. 2014;12:1-10.

26. Hanley JA, McNeil BJ. The Meaning and Use of the Area under a Receiver Operating Characteristic (ROC) Curve. Radiology. 1982;143:29-36. http://www.med.mcgill.ca/epidemiology/hanley/software/Hanley_McNeil_Radiology_82.pdf.

27. Sachdev PS, Blacker D, Blazer DG, Ganguli M, Jeste D V., Paulsen JS, et al. Classifying neurocognitive disorders: The DSM-5 approach. Nat Rev Neurol. 2014;10:634-42. doi:10.1038/nrneurol.2014.181.

28. Collingwood C, Paddick SM, Kisoli A, Dotchin CL, Gray WK, Mbowe G, et al. Development and communitybased validation of the IDEA study Instrumental Activities of Daily Living (IDEA-IADL) questionnaire. Glob Health Action. 2014;7.

29. Dennis M, Kadri A, Coffey J. Depression in older people in the general hospital: A systematic review of screening instruments. Age Ageing. 2012;41:148-54.

30. Wagner AK, Wyss K, Gandek B, Kilima M, Lorenz S, Whiting D. A Kiswahili version of the SF-36 Health Survey for use in Tanzania: translation and tests of scaling assumptions. Qual Life Res. 1999;8:101-10.

31. Lawton MP, Brody EM. Assessment of Older People: Self-Maintaining and Instrumental Activities of Daily Living. Gerontologist. 1969;9 March:179-186.

32. IBM Corp. IBM SPSS Statistics for Windows. 2015.

33. Namdeo SK, Rout SD. Calculating and interpreting Cronbach 's alpha using Rosenberg assessment scale on paediatrician 's attitude and p erception on self esteem. Int J Community Med Public Heal. 2016;3:1371-4.

34. Tavakol M, Dennick R. Making sense of Cronbach's alpha. Int J Med Educ. 2011;2:53-5.

35. Brown JD. Choosing the Right Type of Rotation in PCA and EFA. JALT Testing \& Evaluation SIG Newsletter. 2009;13 November:20-5. http://hosted.jalt.org/test/PDF/Brown31.pdf.

36. Florkowski CM. Sensitivity, specificity, receiver-operating characteristic (ROC) curves and likelihood ratios: Communicating the performance of diagnostic tests. Clin Biochem Rev. 2008;29 Suppl 1 August:S83-7.

37. Wong A, Law LSN, Liu W, Wang Z, Lo ESK, Lau A, et al. Montreal cognitive assessment: One cutoff never fits all. Stroke. 2015;46:3547-50.

38. Kravitz E, Schmeidler J, Schnaider Beeri M. Cognitive Decline and Dementia in the Oldest-Old. Rambam Maimonides Med J. 2012;3:e0026.

39. Jung Kim B, Liu L, Cheung C, Ahn J. Effects of cognitive impairment and functional limitation on depressive symptoms among community-dwelling older Korean immigrants in the U.S. PLoS One. 2018;13:1-9.

40. Zeng Q, Dong X, Ruan C, Hu B, Zhou B, Xue Y, et al. Cognitive impairment in Chinese IIDDs revealed by MoCA and P300. Mult Scler Relat Disord. 2017;16 February:1-7. doi:10.1016/j.msard.2017.05.006. 
41. Spitzer H, Rwegoshora H, Mabeyo ZM. The (Missing) Social Protection for Older People in Tanzania: A Compative Study in Rural and Urban Areas. 2009. https://www.ageing.ox.ac.uk/files/Social Protection Older People Tanzania January 2010.pdf.

42. Kilama B. Where are the poor: Region and District Poverty Estimates for Tanzania, 2012. 2016. http://www.tzdpg.or.tz/fileadmin/documents/external/national_development_frameworks/Poverty_Mapping_PED_June_2016_.pdf.

43. National Bureau of Statistics and Office of Chief Government Statistician. 2012 Population and Housing Census - Basic Demographic and Socio- Economic Profile - Key Findings. Dar es Salaam, Tanzania; 2014.

\section{Tables}

Table 1. Demographic characteristics of the sample and their scores in the neuropsychological tests

\begin{tabular}{|c|c|c|c|c|}
\hline Characteristic & $\begin{array}{l}\text { All participants } \\
\qquad(\mathrm{N}=202)\end{array}$ & $\begin{array}{c}\text { Men } \\
(\mathrm{n}=56,(27.7 \%))\end{array}$ & $\begin{array}{c}\text { Women } \\
(n=146,(72.3 \%))\end{array}$ & $P$ Value \\
\hline Age (Mean, SD) & $73.6(8.0)$ & $76.8(7.2)$ & $72.3(8.0)$ & $<0.001$ \\
\hline Years of Education (Median, Range) & $1(0-17)$ & $4.0(0-17)$ & $0.0(0-12)$ & $<0.001$ \\
\hline \multicolumn{5}{|l|}{ Education by category (n, (\%)) } \\
\hline$\S$ No education & $94(46.5)$ & $12(12.8)$ & $82(87.2)$ & \\
\hline$\S 1$ to 4 years & $79(39.1)$ & $30(38.0)$ & $49(62.0)$ & \\
\hline$\S 5-7$ years & $21(10.4)$ & $9(42.9)$ & $12(57.1)$ & \\
\hline$\S 8$ years or above & $8(4.0)$ & $5(62.5)$ & $3(37.5)$ & \\
\hline GDS-SF [0-15] (Median, Range) & $4(0-14)$ & $4(1-12)$ & $5(0-14)$ & 0.651 \\
\hline MHI-5 score [0-100] (Median, Range) & $76(20-100)$ & $80(24-100)$ & $72(20-100)$ & 0.102 \\
\hline Lawton IADL Score [0-8] (Median, Range) & $7(1-8)$ & $70(3-8)$ & $7(2-8)$ & 0.391 \\
\hline IDEA cognitive screen [0-15] (Mean, SD) & $11.1(2.7)$ & $11.8(2.4)$ & $10.9(2.7)$ & 0.032 \\
\hline MoCA-5-min total [0-30] (Mean, SD) & $19.1(4.5)$ & $20.3(4.5)$ & $18.7(4.4)$ & 0.019 \\
\hline
\end{tabular}

Table 2. The construct validity of the MoCA-5-min: the association of the predictors on MoCA-5-min total scores in the multivariate linear regression model

\begin{tabular}{lccc}
\hline \multicolumn{1}{c}{ Predictor } & $\boldsymbol{\beta}$ & 95\% CI & P value \\
\hline Age & -0.15 & $-0.22-0.07$ & $<0.001$ \\
Years of education & 0.66 & $0.48-0.85$ & $<0.001$ \\
Lawton-IADL scores & 0.41 & $-0.01-0.83$ & 0.054
\end{tabular}


Table 3. Differences in the MoCA-5-min mean scores among the three groups categorised by DSM-V criteria

\begin{tabular}{ccccc}
\hline & Normal Controls & MCI & Dementia & P - trend \\
\hline MoCA-5min (M, SD) & $23.9(3.1)$ & $18.5(3.8)$ & $12.1(3.9)$ & $<0.001$ \\
\hline
\end{tabular}

Note: The post-hoc p-values for group differences were such that: normal controls vs $\mathrm{MCI},<0.001$; normal controls vs dementia, $<0.001$, MCI vs dementia; $<0.001$

Table 4. Areas under the receiver operating characteristics curves showing the power of MoCA-5-min to discriminate mild cognitive impairment from normal cognition across subsamples of different age groups and levels of education.

\begin{tabular}{lccccc}
\hline \multicolumn{1}{c}{ Years of education } & AUC & P value & Optimal cut-off & Sensitivity & Specificity \\
\hline Age Groups & & & & & \\
§ Early older adults ${ }^{\mathrm{a}}$ & 0.85 & $<0.001$ & 23 & $75 \%$ & $80 \%$ \\
§ Middle older adults ${ }^{\mathrm{b}}$ & 0.87 & $<0.001$ & 21 & $80 \%$ & $80 \%$ \\
§ Oldest old & 0.78 & 0.063 & $18 / 19$ & $78 \%$ & $75 \%$ \\
Levels of education & & & & & \\
§ No education & $\mathrm{NV}$ & - & - & - & - \\
§ Any education & 0.83 & $<0.001$ & $22 / 23$ & $75 \%$ & $74 \%$ \\
§ 1 - 4 & 0.81 & $<0.001$ & $22 / 23$ & $76 \%$ & $75 \%$ \\
§5 years or more & 0.80 & 0.011 & $23 / 24$ & $78 \%$ & $63 \%$ \\
\hline
\end{tabular}

Note. AUC, area under the receiver operating characteristics curve; ${ }^{a}$, aged 60 - 69 years; ${ }^{b}$, aged $70-79$ years; ${ }^{c}$, aged 80 years or older; NV, the ROC analysis was not valid, there was only one normal cognitive control in the group for comparative analysis.

\section{Figures}




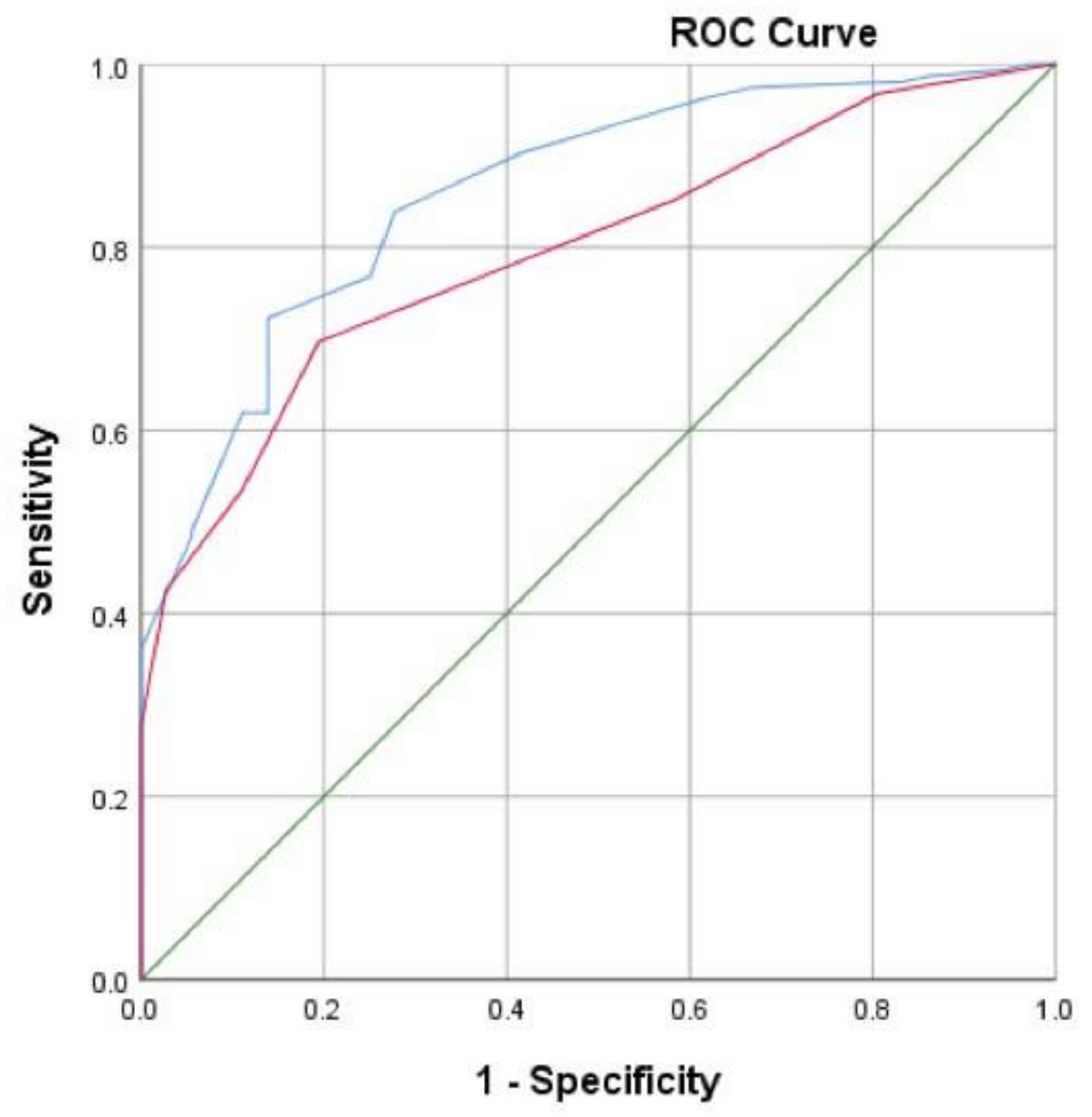

Source of the Curve

MoCA-5-min_Total Score IDEA_Global score Reference Line

Figure 1

The ROC curve of MoCA-5-min and IDEA cognitive screen in detecting $\mathrm{MCl}$ 


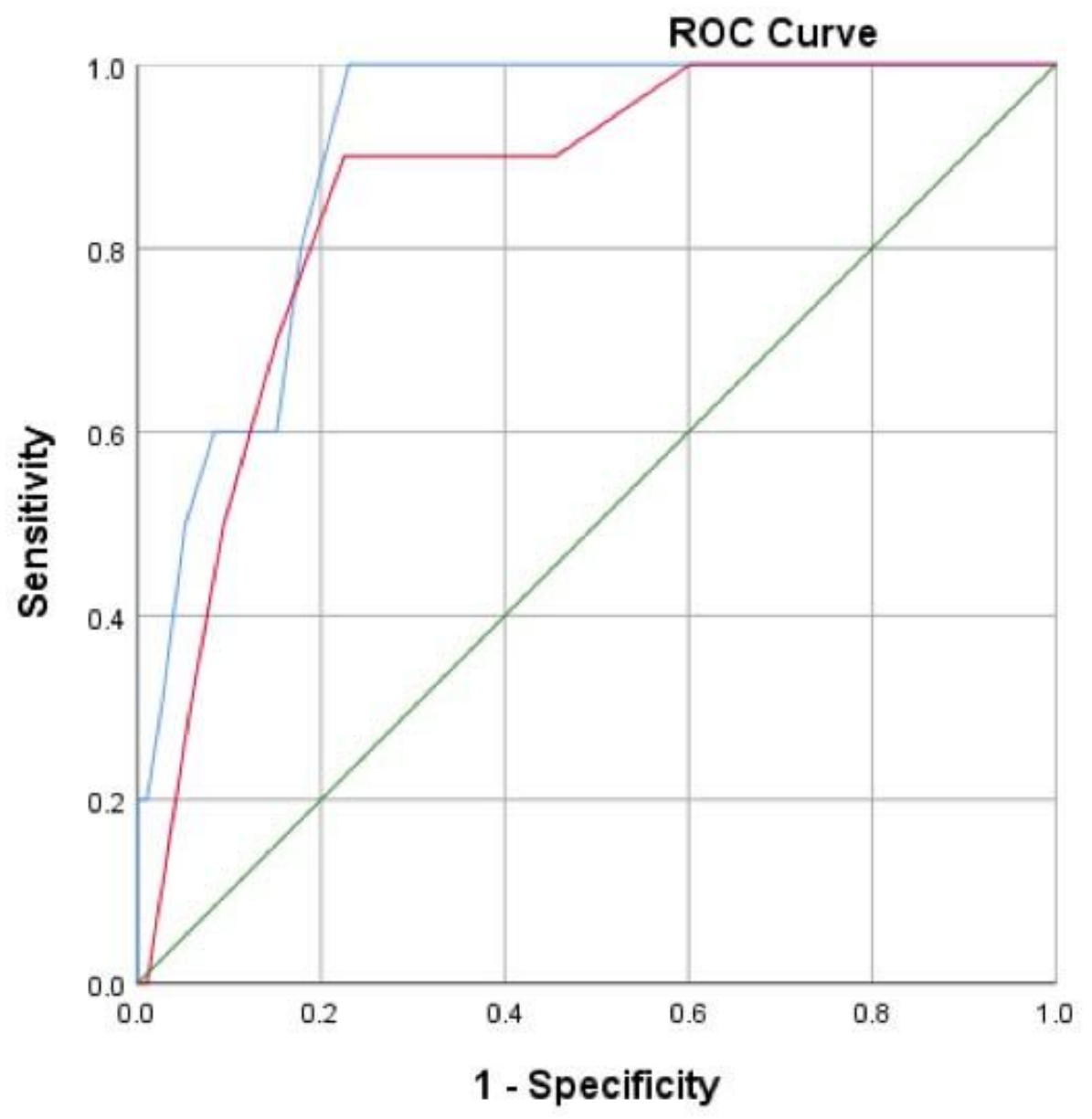

\section{Source of the Curve}

MoCA-5-min_Total Score IDEA_Global score Reference Line

\section{Figure 2}

The ROC curve of MoCA-5-min and IDEA cognitive screen in detecting dementia 